\title{
An improved simulated annealing algorithm based on residual network for permutation flow shop scheduling
}

\author{
Yang $\mathrm{Li}^{1} \cdot$ Cuiyu Wang ${ }^{1} \cdot$ Liang Gao $^{1} \cdot$ Yiguo Song ${ }^{2} \cdot$ Xinyu $\mathrm{Li}^{1}$
}

Received: 30 June 2020 / Accepted: 18 September 2020 / Published online: 8 October 2020

(c) The Author(s) 2020

\begin{abstract}
The permutation flow shop scheduling problem (PFSP), which is one of the most important scheduling types, is widespread in the modern industries. With the increase of scheduling scale, the difficulty and computation time of solving the problem will increase exponentially. Adding the knowledge to intelligent algorithms is a good way to solve the complex and difficult scheduling problems in reasonable time. To deal with the complex PFSPs, this paper proposes an improved simulated annealing (SA) algorithm based on residual network (SARes). First, this paper defines the neighborhood of the PFSP and divides its key blocks. Second, the Residual Network (ResNet) is used to extract and train the features of key blocks. And, the trained parameters are stored in the SA algorithm to improve its performance. Afterwards, some key operators, including the initial temperature setting and temperature attenuation function of SA algorithm, are also modified. After every new solution is generated, the parameters trained by the ResNet are used for fast ergodic search until the local optimal solution found in the current neighborhood. Finally, the most famous benchmarks including part of TA benchmark are selected to verify the performance of the proposed SARes algorithm, and the comparisons with the-state-of-art methods are also conducted. The experimental results show that the proposed method has achieved good results by comparing with other algorithms. This paper also conducts experiments on network structure design, algorithm parameter selection, CPU time and other problems, and verifies the advantages of SARes algorithm from the aspects of stability and efficiency.
\end{abstract}

Keywords Permutation flow shop scheduling $\cdot$ Improved simulated annealing algorithm $\cdot$ Residual networks $\cdot$ TA benchmark

\section{Introduction}

Scheduling is an indispensable part of the modern manufacturing process. Intelligent workshop scheduling can not only ensure the orderly progress of workshop manufacturing process but also maximize the utilization of resources and reduce the waste in the manufacturing process, thus reducing the production and manufacturing cost [28]. The permutation flow shop scheduling problem (PFSP), which is one of the most important scheduling types, is widespread in the modern industries, including automobile [35], electronic [5], chemical [20] and other industries. Therefore, the effective

Xinyu Li

lixinyu@mail.hust.edu.cn

1 The State Key Laboratory of Digital Manufacturing Equipment and Technology, Huazhong University of Science and Technology, Wuhan, China

2 The State Key Laboratory of CAD\&CG, Zhejiang University, Hangzhou, China
PFSP algorithm can improve the productivity of these industries well.

However, the PFSP is a well-known NP-hard problem and is very hard to be solved in large scales. At present, most of the PFSP methods mainly focus on the meta-heuristic algorithms, such as genetic algorithm (GA) [30], simulated annealing algorithm (SA) [10], tabu search algorithm (TS) [11], particle swarm optimization algorithm (PSO) [39], etc. In recent years, there have been some related research works. Various meta-heuristic algorithms emerge one after another, which provides a lot of theoretical basis for the study of PFSP [29, 31, 32]. Suresh et al. [25] proposed a social group optimization (SGO), which is a population-based optimization technique. Taking advantage of both epsilon greedy and Levy flight, Liu et al. [16] proposed a greedy-Levy Ant colony optimization (ACO) incorporating these two approaches to solve the complicated combinatorial optimization problems. Sayed et al. [22] presented a hybrid algorithm based on moth-flame optimization (MFO) algorithm with simulated annealing (SA), namely (SA-MFO). It can escape from local 
optima mechanism of SA and fast searching and learning mechanism for guiding the generation of candidate solutions of MFO.

Although these meta-heuristic algorithms have some advantages, the solution quality may be poor and the computation time may be long when dealing with the large-scale and complex PFSPs [7]. Because, the previous historical data cannot be used to mine the variation rules of scheduling, especially for large-scale and complex problems, which may fail to achieve good results, and the calculation time will increase exponentially [13]. Therefore, to enhance the optimization capability when solving complex problems, it is very important to incorporate the knowledge into the algorithm. Adding the knowledge to intelligent algorithms is a good way to solve the complex and difficult scheduling problems in reasonable time, including the PFSPs [18]. Therefore, this paper adopts a knowledge-driven method to assist the intelligent algorithm for the PFSPs.

But, how to extract the knowledge from the historical data is a very challenging work. Deep learning is a very effective method to extract knowledge based on historical data and some prior knowledge to form its own knowledge system and learning skills. Yoshua et al. [37] surveys the recent attempts, both from the machine learning and operations research communities, at leveraging machine learning to solve the combinatorial optimization problems.

Therefore, this paper uses the residual networks (ResNet), a very good and simple deep learning method, as a tool to train and classify the features for the PFSPs. By quickly judging the neighborhood of the problem, the intelligent algorithm can traverse as many solutions as possible in a very short time. Because the network training process can precede the start of scheduling, precious scheduling time is not consumed. This way can not only improve the effectiveness of the algorithm but also reduce the computation time.

As a kind of convolutional neural networks ( $\mathrm{CNN})$, ResNet is one of the most commonly used deep learning methods. In 2015, He et al. [8] used ResNet to solve the problem of gradient disappearance. Valeryi et al. [26] proposed the technique of automatic labeling the data set with the finite element model for training of artificial neural network in tomography. Lin et al. [15] proposed a residual networks of residual networks (RoR) optimization method to avoid over-fitting and gradient vanish. Gomez et al. [6] found in the ResNet network along with the increase of the depth in improving performance, but increased memory consumption, thus put forward each layer's activations can be reconstructed exactly from the next layer's. Li et al. [14] propose a multi-scale residual network to solve the problem of super-resolution in different scales of a single image. Zhong et al. [40] inspired by the residual network, designed an endto-end spectral-spatial residual network (SSRN) that takes raw 3-D cubes as input data without feature engineering for hyperspectral image classification.

The main feature of ResNet is the cross-layer connectivity, where can transfer inputs across layers and add to the results of convolution by introducing shortcut connections. Deep neural networks are very difficult to train [19]. The ResNet proposes a framework for learning residuals to simplify the training of networks that are deeper than those previously used. Instead of learning unknown functions, it has explicitly defined learning residual functions. Comprehensive empirical evidences indicate that the ResNet is easier to optimize and its accuracy can be obtained with significantly increased depth. There is only one pooling layer in ResNet, which is connected behind the last convolutional layer. ResNet enables the underlying network to be fully trained, and the accuracy rate is significantly improved with the deepening of the depth. ResNet, with a depth of 152 layers, won first place in the 1SVRC-15 image classification competition. With its good performance, this paper uses it to classify the extracted workshop characteristic data. According to the classification results, it can quickly judge whether the current neighborhood search operation is effective or not.

Since the ResNet assisted method proposed in this paper can be used to improve the most meta-heuristic algorithms, to verify its effectiveness, an improved SA algorithm based on ResNet (SARes) is proposed for PFSP in this paper. The most famous benchmarks of PFSP including part of TA benchmark are selected to verify the performance of the proposed algorithm, and the comparisons with the-state-of-art methods are also conducted. The experimental results show that the proposed SARes method has achieved significant results. This paper also conducts experiments on network structure design, algorithm parameter selection, CPU time and other problems, and verifies the advantages of SARes algorithm from the aspects of stability and efficiency.

The rest of this paper is organized as follows: Sect. 2 introduces the PFSP. Section 3 provides the proposed SARes for PFSP. Section 4 gives the experimental results and comparisons with the-state-of-art algorithms. The final section shows the conclusion and future work.

\section{Problem formulation}

\section{Introduction of permutation flow shop scheduling problem}

In 1954, Johnson [9] first proposed and studied the PFSP. Since then, PFSP has attracted the attentions from a large number of scholars. After decades of research, a lot of good algorithms had been proposed to solve such problems [27].

In the PFSP, the solutions are represented by the permutation of $n$ jobs, i.e., $\sigma=\left\{\sigma_{1}, \sigma_{2}, \ldots, \sigma_{n}\right\}$. Each job contains 
$m$ operations, and every operation is performed by a different machine. Jobs, once initiated, cannot be interrupted (preempted) by another job on each machine and the release time of all jobs is zero. Thus, given the processing time $t_{j k}$ for the job $j$ on the machine $i$, the PFSP is to find the best permutation of jobs $\sigma^{*}=\left\{\sigma_{1}^{*}, \sigma_{2}^{*}, \ldots, \sigma_{n}^{*}\right\}$ to be processed on each machine to optimize the objectives, such as the makespan. $C\left(\sigma_{j}, m\right)$ denotes the completion time of the job $\sigma_{j}$ on the machine $m$ [18].

Then given the job permutation $\sigma$, the completion time for the $n$-job, $m$-machine problem is calculated as follows:

$$
\left\{\begin{array}{c}
C_{\sigma_{1}, 1}=t_{\sigma_{1}} \\
C_{\sigma_{1}, j}=C_{\sigma_{1}, j-1}+t_{i j}, \quad j=2,3, \ldots, m \\
C_{\sigma_{i}, 1}=C_{\sigma_{i-1}, 1}+t_{i 1}, \quad i=2,3, \ldots, n \\
C_{\sigma_{i}, j}=\max \left(C_{\sigma_{i-1}, j}, C_{\sigma_{i}, j-1}\right)+t_{i j}, \\
i=2,3, \ldots, n ; \quad j=2,3, \ldots, m
\end{array}\right.
$$

$t_{i j}$ represents the processing time of job $i$ on machine $j$. The best solution is with the minimum makespan.

Although the PFSP is a special type of flow shop scheduling, it is still a very complex combinatorial optimization problem [36]. With the increase of jobs, the size of the solution space will increase exponentially, so it is extremely difficult to find a satisfactory solution for the large scale PFSPs.

\section{Definition of neighborhood for PFSP}

In this study, the neighborhood search of PFSP is defined as follows: Exchange the machining order of all adjacent jobs in turn, update if the solution quality becomes better, otherwise continue to exchange the next set of jobs. Until any two adjacent jobs in the sequence are swapped and the result cannot be improved, the local optimal solution under the current neighborhood is obtained by default.

As shown in Fig. 1, this is the Gantt chart of the PFSP. Suppose we currently need to determine whether the new solution after exchanging jobs 8 and 5 has been improved. The traditional approach is to recalculate the makespan as a whole. This will take a lot of time, which will affect the final solution efficiency.

The analysis of Gantt chart shows that the order of the two sides of the red area does not change. Therefore, the results can only be judged by the changes in the red area. For the example given in the figure, the result of swapping 8 and 5 is only related to the end of 9 , the beginning of 8 and 5 , the end of 8 and 5, and the beginning of 11 . In this paper, the red areas are defined as key blocks.

If we can find the relationship, we can quickly determine the quality of the solution and reduce the computation time.
However, it is difficult to find relevant laws artificially, so artificial intelligence is needed.

\section{Proposed SARes for PFSP}

According to the analysis of the current situation of the PFSP problem, the combination explosion problem must be dealt with before the problem to be solved. When the scale of the problem grows, the ergodic solution takes too long to get the result. However, algorithms with stochastic properties such as meta-heuristic may fall into the local optimal solutions. Therefore, the knowledge-driven approach is adopted to speed up the search process as much as possible, so that the meta-heuristic can search more neighborhoods within a limited time.

\section{Introduction of ResNet}

The basic structure of CNN is composed of the input layer, the convolutional layer, the pooling layer (also known as the sampling layer), the full connection layer and the output layer. It has the structural characteristics of local area connection, weight sharing and down sampling. Its development has gone through several critical periods:

In 2015, He et al. [8] used residual networks (ResNet) to solve the problem of gradient disappearance. The main feature of ResNet is cross-layer connectivity, where you transfer inputs across layers and add to the results of convolution by introducing shortcut connections.

Deep network has the problem of gradient disappearance or explosion, which makes deep learning model difficult to train. The degradation of the deep network at least indicates that deep network is not easy to train. So, ResNet is used to solve this problem.

The structure of the residual block is shown in Fig. 2. In general network structure, the input $x_{1}$ directly passes through two convolutional layers to obtain the output $x_{1}+$ 1 , while the residual block is to add the output through two convolutional layers to the network input $x_{1}$. We can call this method as skip connection. Skip Connection is not only allowed to add the input $x_{1}$ and convolution results directly. In some cases, the input $x_{1}$ and $x_{1}+1$ dimension are different, so $1 * 1$ convolution can be added to reduce the dimension of the input so that $x_{1}$ and $x_{1}+1$ dimension are the same, and the two can be added.

Suppose the input is $X$, and there are two fully bonded layers that asymptotically fit $H(x)$. Assuming that $H(x)$ is the same as $\mathrm{x}$ dimension, then fitting $H(x)$ is equivalent to fitting residual function $H(x)-x$. If the residual function $F(x)=H(x)-x$, then the original function becomes $F(x)$ $+x$. Then a cross-layer connection is directly added on the

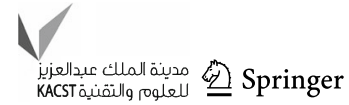


Fig. 1 The Gantt chart of the PFSP

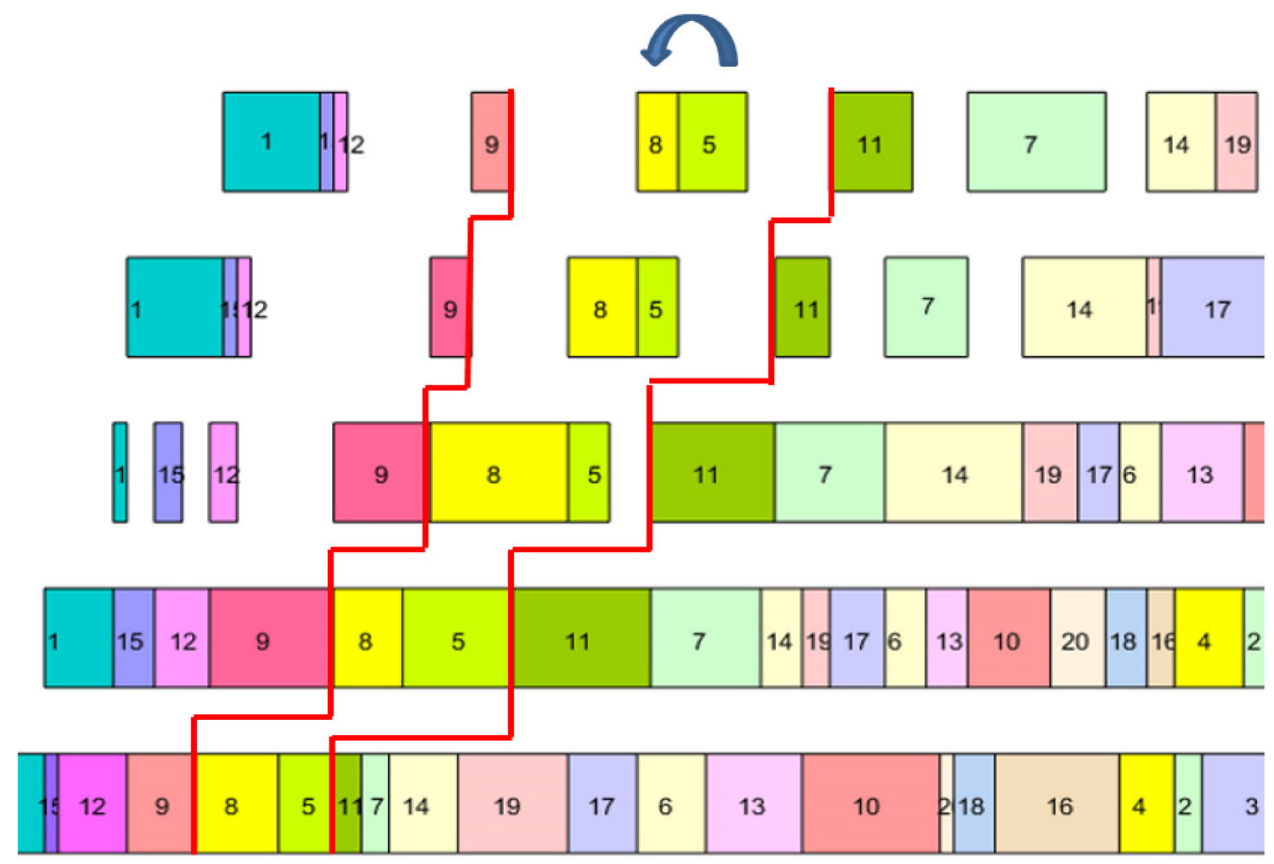

Fig. 2 Residual learning: a building block

basis of the original network, and the cross-layer connection here is to transfer $X$ 's Identity Mapping.

The entire residual structure can be formally defined as:

$y=F(x,\{W i\})+x$.

Here $F(x,\{W i\})$ refers to the fitting residual mapping, as shown in the Fig. 2, there are two full connection layers, $F=$ $W_{2} \sigma\left(W_{1} x\right)$, where $\sigma$ refers to ReLU. When $F$ and $x$ have the same dimension, they can be added to each element directly. But if not, we have to add a linear map to $x$ and map it to a vector that has the same dimension as $F$, so the whole residual structure is:

$y=F(x,\{W i\})+W_{s} x$.

$W_{s}$ is a matrix for dimensional matching.

\section{Combination of residual network and scheduling}

According to the above introduction, the ResNets is mainly used to solve the image classification. For scheduling, we can think of the key block matrix as an image. First, we solve the makespan directly and annotate the critical blocks. Then, a large number of labeled key blocks are used as training sets to train the residual network. Finally, the trained residual network parameters are saved and used to judge the influence of the processing order of adjacent jobs under the same scale on the result. If the network output is "better", then we swap the current jobs. If the network output is "worse", the original sequence is left unchanged and takes place the next iteration.

The training data used in this paper are self-generated. For the case of the same scale, the key blocks of the sequence are tagged by the traditional method and the tagged samples were used as training sets for ResNet.

Taking Fig. 1 as an example, the operation process is shown in Fig. 3.

\section{Improved simulated annealing algorithm for PFSP}

The SA algorithm originated from the Metropolis criterion proposed by Metropolis et al. in 1953 [1]. Many studies have shown that SA algorithm is a good optimization algorithm that can converge to the optimum solution with probability 1 . In 1983, Kirkpatrick et al. successfully introduced the idea of annealing into the optimization field [10]. SA has been widely used in various optimization problems due to its strong local searching ability, easy operation and fast solving speed. In this paper, some improvements are made to the SA algorithm. 


\begin{tabular}{|c|c|c|c|c|c|c|}
\hline job & 9 & \multicolumn{2}{|c|}{8} & \multicolumn{2}{|c|}{5} & 11 \\
\hline machine & End & Star & End & Star & End & Star \\
\hline 1 & 573 & 573 & 641 & 641 & 695 & 695 \\
\hline 2 & 659 & 659 & 664 & 695 & 774 & 774 \\
\hline 3 & 734 & 734 & 811 & 811 & 827 & 873 \\
\hline 4 & 811 & 811 & 862 & 862 & 928 & 928 \\
\hline 5 & 865 & 865 & 933 & 933 & 991 & 996 \\
\hline
\end{tabular}

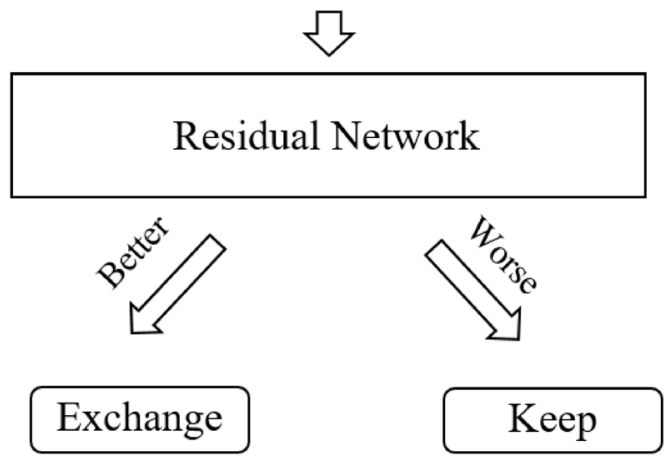

Fig. 3 Residual network operation flow chart

\section{Encoding and parameter initialization}

Encoding method adopts natural number coding: $n$ jobs are numbered $1-n$ successively. The generated scheduling scheme is consistent with the corresponding natural number coding. The initial solution is generated by NEH algorithm [24]. In this paper adopts the method of random generation of a set of state, to determine the difference between the two states biggest target "I $\Delta$ maxl", then according to the difference value, the initial acceptance probability is used to determine the initial temperature [38]. The relevant expression is as follows:

$T_{0}=-\Delta \max / p_{0}$.

Based on computational experience, the parameters are set as follows: $k=2000, a=0.95, P_{0}=0.2$. Among them, $k$ is markov chain length, $a$ is temperature attenuation coefficient and $P_{0}$ is the initial acceptance probability.

\section{Multi-rule neighborhood search}

The neighborhood has been defined in the Sect. 2, and it is proved that the local optimal solution can be found in the current neighborhood. Therefore, in the process of iteration, we need to disrupt the original sequence as much as possible to make it jump out of the local optimal solution. In this paper, three ways of generating new solutions are selected randomly according to a certain probability.
1. Binary exchange: randomly select two points in the coding sequence and reverse the order of all the jobs between them.

2. Three points exchange: randomly select three points in the coding sequence, and exchange two sequence positions between them.

3. Two points exchange: randomly select two points in the coding sequence and exchange the job positions of these two points.

For each new solution generated, the method given above is used to search through ergodic in the neighborhood until the local optimal solution under the current neighborhood is found. At the same time, to ensure that excellent solutions are not replaced in subsequent operations, we set a global variable E_best to hold the optimal solution.

\section{Temperature decay function}

The temperature attenuation function is an important parameter that affects the performance of simulated annealing algorithm [4]. The formula of Kepler-type decay function decline curve is as follow:

$$
\begin{gathered}
T=T_{0} a^{k}(\cos (\pi /(2(1-k / K))) \\
+\cos \left(\pi /\left(2 T_{0}(1-k / K)\right),\right.
\end{gathered}
$$

$a$ is temperature attenuation coefficient, $k$ is the number of iterations, and $K$ is the total cooling times. This function is executed every time the neighborhood search is completed until the temperature $T$ reaches the minimum. After the temperature attenuation is completed, the optimal objective function value is returned and the program is finished. In each decay. If 200 consecutive iterations fail to update the current optimal solution, the search at the current temperature is ended.

\section{Framework of the improved SARes}

The improved simulated annealing algorithm is shown in the Fig. 4. Driven by knowledge, the meta-heuristic algorithm increases the stable global searching capability on the basis of randomness. In each iteration at temperature, the optimal solution in the current search space can be obtained. At the same time, the learning and training time of the algorithm can be completed in advance without occupying the scheduling time, which greatly increases the overall computing efficiency of the algorithm.

Here is an example of how to combine ResNet and SA in PFSP. Suppose there are five machines and ten jobs in the factory. First, we numbered them 1 to 10. Then, the generated initial sequence was input into the SA for iteration. After each

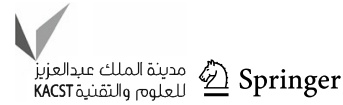




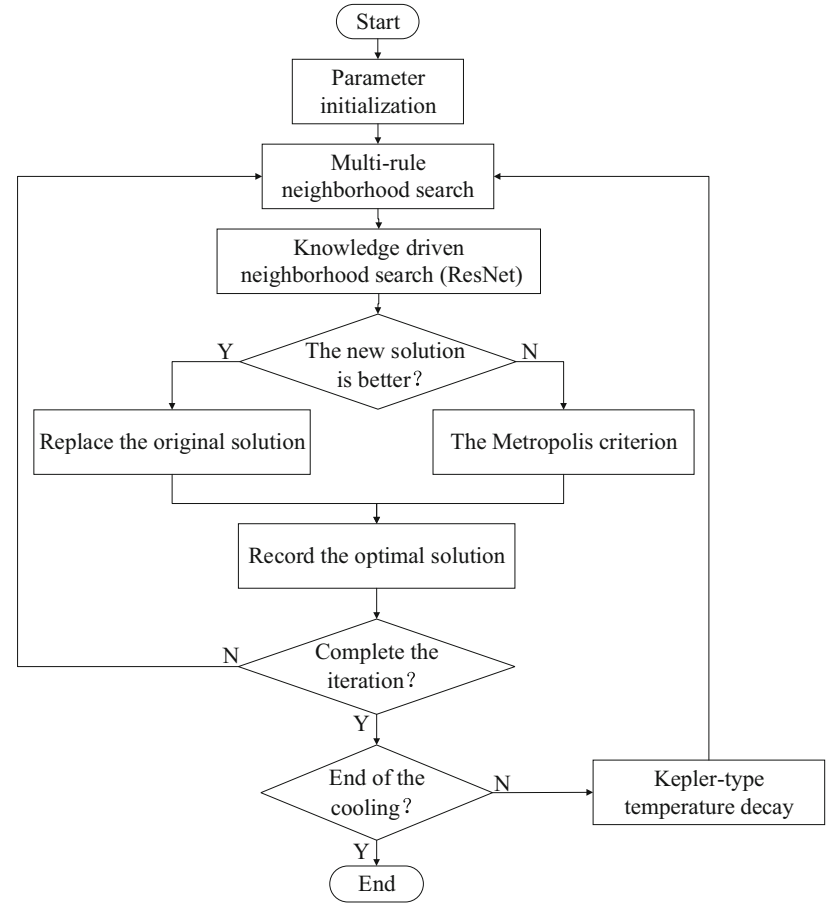

Fig. 4 Flow chart of the proposed SA algorithm

neighborhood search described in Sect. 3.3.2 is performed, the resulting sequence is imported into the trained ResNet. Assume that the sequence at this time is $(5,4,3,6,8,7,9$, 2, 1). Next, the neighborhood search described in Sect. 3.2 begins. We need to judge whether the exchanging position of all adjacent jobs (such as 5 and 4, 4 and 3, 3 and 6) can make the result better. To make a judgment quickly, we took the key blocks in Sect. 2.2 as input and put them into the trained Resnet to get the final judgment result. Adjust the sequence according to the result until all adjacent jobs are exchanged to make the result better. With this result as the local optimal solution at the current SA temperature, restart the execution of the SA program. After the final execution of SA, the scheduling scheme under the current workshop can be obtained.

\section{Experimental results and discussion}

\section{The experimental setup}

The proposed algorithm is encoded in python and runs on a laptop with the following capabilities: Intel Core i7-8750H CPU @ 2.20 GHz with 16.0 GB Memory in Windows 10.

To facilitate the performance of the algorithm, the evaluation index includes makespan, the average relative percentage deviation (ARPD).
$\mathrm{ARPD}=\frac{\left(\sum_{i=1}^{n} C_{i}\right) / n-\mathrm{UB}}{\mathrm{UB}} \cdot 100 \%$,

$n$ is the running times of the algorithm, $C_{i}$ is the result of the $i$ th running of the algorithm, and UB is the currently known best solution.

This paper selects the part of TA benchmark (number of jobs $\geq 100$ ) to evaluate the performance of the proposed algorithm. TA benchmark is one of the most famous benchmarks, which contains the test data of various workshop scheduling models including PFSP [3].

Because when the number of jobs is less than 100 , most algorithms can find the optimal solution. Therefore, this paper selects cases with more than 100 jobs for the comparative experiment (TA61-TA120). This paper also conducts experiments on network structure design, algorithm parameter selection, CPU time and other problems, and verifies the advantages of SARes algorithm from the aspects of stability and efficiency.

\section{Experimental results of Taillard benchmark}

Taillard [26] proposed TA benchmark in 1993, which contains 260 different workshop scheduling instances. It became one of the most widely used benchmarks in the field of scheduling. All problem determination of processing time and the known optimal solution can refer to https://mistic.heigvd.ch/taillard/problemes.dir/ ordonnancement.dir/ordonnancement.html.

The selected state-of-the-art comparison algorithms are as follows:

- Self-guided differential evolution with neighborhood search (NS-SGDE, 2016) [23];

- Hybrid differential evolution (L-HDE, 2014) [33];

- Hybrid teaching learning-based optimization (HTLBO, 2014) [34];

- Estimation of distribution algorithm variable neighborhood search (EDA-VNS, 2017) [17];

- Opposition-based differential evolution (ODDE, 2013) [12];

- Extended artificial chromosomes genetic algorithm (eACGA, 2012) [2].

For each instance in the benchmark, run it 10 times and record the results, then calculate the ARPD according to the formula (6). The results of Taillard benchmark are shown in Table 1. Data statistics are shown in Table 2. The significance of bold is the best solution on the same scale in the table. Because some references (L-HDE, HTLBO, EDA-VNS) did not provide the specific makespan for every instance, only some algorithms (SARes, ISA, NS-SGDE, ODDE, eACGA) are compared in Table 1. Among them, "ISA" represents the 
Table 1 Comparison on Taillard benchmark based on the best scheduling

\begin{tabular}{|c|c|c|c|c|c|c|}
\hline Instance & UB & SARes & ISA & NS-SGDE [23] & ODDE [12] & eACGA [2] \\
\hline TA61 & 5493 & 5493 & 5493 & 5493 & 5493 & 5493 \\
\hline TA62 & 5268 & 5283 & 5284 & 5283 & 5284 & 5284 \\
\hline TA63 & 5175 & 5175 & 5175 & 5182 & 5185 & 5177 \\
\hline TA64 & 5014 & 5018 & 5023 & 5018 & 5018 & 5018 \\
\hline TA65 & 5250 & 5250 & 5250 & 5253 & 5253 & 5253 \\
\hline TA66 & 5135 & 5135 & 5135 & 5135 & 5135 & 5135 \\
\hline TA67 & 5246 & 5246 & 5246 & 5246 & 5246 & 5246 \\
\hline TA68 & 5094 & 5094 & 5112 & 5094 & 5095 & 5098 \\
\hline TA69 & 5448 & 5448 & 5448 & 5448 & 5454 & 5454 \\
\hline TA70 & 5322 & 5322 & 5346 & 5322 & 5322 & 5328 \\
\hline TA71 & 5770 & 5780 & 5780 & 5784 & 5800 & 5783 \\
\hline TA72 & 5349 & 5362 & 5403 & 5362 & 5378 & 5367 \\
\hline TA73 & 5676 & 5691 & 5693 & 5691 & 5698 & 5679 \\
\hline TA74 & 5781 & 5826 & 5830 & 5826 & 5890 & 5848 \\
\hline TA75 & 5467 & 5491 & 5491 & 5503 & 5533 & 5512 \\
\hline TA76 & 5303 & 5308 & 5317 & 5308 & 5309 & 5317 \\
\hline TA77 & 5595 & 5610 & 5628 & 5610 & 5633 & 5618 \\
\hline TA78 & 5617 & 5630 & 5687 & 5630 & 5695 & 5660 \\
\hline TA79 & 5871 & 5882 & 5940 & 5882 & 5940 & 5926 \\
\hline TA80 & 5845 & 5881 & 5903 & 5881 & 5896 & 5881 \\
\hline TA81 & 6202 & 6310 & 6339 & 6360 & 6458 & 6407 \\
\hline TA82 & 6183 & 6268 & 6317 & 6278 & 6364 & 6349 \\
\hline TA83 & 6271 & 6384 & 6391 & 6405 & 6493 & 6421 \\
\hline TA84 & 6269 & 6349 & 6364 & 6394 & 6461 & 6410 \\
\hline TA85 & 6314 & 6421 & 6439 & 6452 & 6535 & 6475 \\
\hline TA86 & 6364 & 6455 & 6487 & 6461 & 6603 & 6511 \\
\hline TA87 & 6268 & 6372 & 6382 & 6385 & 6463 & 6441 \\
\hline TA88 & 6401 & 6496 & 6549 & 6496 & 6641 & 6580 \\
\hline TA89 & 6275 & 6362 & 6384 & 6428 & 6501 & 6458 \\
\hline TA90 & 6434 & 6562 & 6575 & 6538 & 6616 & 6575 \\
\hline TA91 & 10,862 & 10,887 & 10,914 & 10,887 & 10,933 & 10,928 \\
\hline TA92 & 10,480 & 10,517 & 10,531 & 10,555 & 10,570 & 10,573 \\
\hline TA93 & 10,922 & 10,962 & 11,000 & 10,980 & 11,006 & 11,017 \\
\hline TA94 & 10,899 & 10,910 & 10,939 & 10,917 & 10,939 & 10,939 \\
\hline TA95 & 10,524 & 10,537 & 10,537 & 10,537 & 10,575 & 10,575 \\
\hline TA96 & 10,326 & 10,338 & 10,388 & 10,357 & 10,409 & 10,395 \\
\hline TA97 & 10,854 & 10,931 & 10,945 & 10,929 & 10,966 & 10,945 \\
\hline TA98 & 10,730 & 10,785 & 10,810 & 10,798 & 10,798 & 10,828 \\
\hline TA99 & 10,438 & 10,467 & 10,495 & 10,465 & 10,505 & 10,473 \\
\hline TA100 & 10,675 & 10,727 & 10,727 & 10,727 & 10,758 & 10,744 \\
\hline TA101 & 11,195 & 11,315 & 11,403 & 11,468 & 11,527 & 11,481 \\
\hline TA102 & 11,203 & 11,412 & 11,432 & 11,487 & 11,646 & 11,634 \\
\hline TA103 & 11,281 & 11,489 & 11,503 & 11,549 & 11,721 & 11,662 \\
\hline TA104 & 11,275 & 11,502 & 11,516 & 11,553 & 11,663 & 11,639 \\
\hline TA105 & 11,259 & 11,459 & 11,459 & 11,438 & 11,578 & 11,533 \\
\hline TA106 & 11,176 & 11,396 & 11,413 & 11,445 & 11,587 & 11,544 \\
\hline TA107 & 11,360 & 11,555 & 11,599 & 11,596 & 11,725 & 11,757 \\
\hline
\end{tabular}


Table 1 continued

\begin{tabular}{llrlllr}
\hline Instance & UB & SARes & \multicolumn{1}{l}{ ISA } & NS-SGDE [23] & ODDE [12] & eACGA [2] \\
\hline TA108 & 11,334 & $\mathbf{1 1 , 5 6 1}$ & 11,581 & 11,592 & 11,699 & 11,692 \\
TA109 & 11,192 & $\mathbf{1 1 , 3 9 3}$ & 11,471 & 11,485 & 11,556 & 11,540 \\
TA110 & 11,288 & $\mathbf{1 1 , 4 8 7}$ & 11,533 & 11,607 & 11,679 & 11,660 \\
TA111 & 26,059 & $\mathbf{2 6 , 3 7 4}$ & 26,442 & 26,420 & 26,639 & 26,763 \\
TA112 & 26,520 & 26,951 & 27,029 & $\mathbf{2 6 , 9 4 2}$ & 27,167 & 27,214 \\
TA113 & 26,371 & $\mathbf{2 6 , 7 2 7}$ & 26,810 & 26,729 & 26,962 & 26,978 \\
TA114 & 26,456 & $\mathbf{2 6 , 7 4 2}$ & 26,790 & 26,751 & 27,027 & 27,022 \\
TA115 & 26,334 & $\mathbf{2 6 , 5 7 9}$ & 26,690 & 26,643 & 26,787 & 26,928 \\
TA116 & 26,477 & $\mathbf{2 6 , 8 1 4}$ & 26,865 & 26,832 & 26,939 & 27,127 \\
TA117 & 26,389 & $\mathbf{2 6 , 5 7 4}$ & 26,790 & 26,609 & 26,754 & 26,931 \\
TA118 & 26,560 & $\mathbf{2 6 , 9 2 5}$ & 27,010 & $\mathbf{2 6 , 9 2 5}$ & 27,107 & 27,173 \\
TA119 & 26,005 & $\mathbf{2 6 , 3 1 1}$ & 26,372 & 26,326 & 26,477 & 26,535 \\
TA120 & 26,457 & $\mathbf{2 6 , 7 6 6}$ & 26,826 & $\mathbf{2 6 , 7 6 6}$ & 26,954 & 26,924 \\
Number of optimal & $\mathbf{5 4}$ & 10 & 26 & 5 & 5 \\
solutions & & & & & & 5 \\
\hline
\end{tabular}

Table 2 Comparison on Taillard benchmark based on the ARPD

\begin{tabular}{|c|c|c|c|c|c|c|c|c|}
\hline Problem & SARes & ISA & NS-SGDE [23] & L-HDE [32] & HTLBO [33] & EDA-VNS [17] & ODDE [12] & eACGA [2] \\
\hline $100 * 5$ & 0.036 & 0.129 & 0.056 & 0.409 & 0.262 & 0.631 & 0.076 & 0.078 \\
\hline $100 * 10$ & 0.330 & 0.704 & 0.358 & 1.306 & 1.218 & 1.216 & 0.877 & 0.559 \\
\hline $100 * 20$ & 1.584 & 1.978 & 1.933 & 4.773 & 3.594 & 1.827 & 3.420 & 2.616 \\
\hline $200 * 10$ & 0.328 & 0.460 & 0.414 & 1.315 & 1.222 & 0.457 & 0.702 & 0.662 \\
\hline $200 * 20$ & 1.782 & 2.032 & 2.360 & 2.972 & 2.880 & 1.815 & 3.392 & 3.179 \\
\hline $500 * 20$ & 1.189 & 1.515 & 1.257 & 1.965 & 1.903 & 1.333 & 1.967 & 2.264 \\
\hline$A R P D$ & 0.875 & 1.136 & 1.063 & 2.123 & 1.847 & 1.213 & 1.734 & 1.560 \\
\hline
\end{tabular}

improved SA algorithm proposed in Sect. 3, which does not include the neighborhood search part. "SARes" represents a new knowledge-driven algorithm based on improved SA.

In the 60 benchmarks shown in Table 1, the knowledgedriven algorithm proposed in this paper achieves 54 optimal solutions, which is far better than other algorithms. Meanwhile, from the average value shown in Table 2, it has the lowest value with 0.875 and outperforms all other algorithms including ISA. It is also evident that the solution space clipping method is effective and reliable when solving PFSPs.

The above test results of TA benchmark show that when solving complex system problems, it is necessary to add knowledge-driven methods to improve the original intelligent algorithm.

It can be seen from the above experiments that the knowledge-driven residual network scheduling model proposed in this paper is effective and the-state-of-art results in TA benchmark. The experimental results show that the knowledge-driven algorithm can solve complex system problems better than the original model.

At the same time, SARes is also a randomized method with certain instability. Therefore, this paper takes TA81 as an example to compare the stability of SARes and ISA. The two methods are used to calculate TA81 for 10 times, respectively, and the resulting figure is shown in Fig. 5. It can be seen from the experiment that the variance of SARes and ISA is 80.27 and 1138.06, respectively. It can be seen from the comparison of Fig. 5 and variance that the SARes method proposed in this paper has a better stability.

\section{Calculation time comparison}

Since the training process of a neural network can be completed in advance and does not occupy the scheduling time, it does not affect the overall efficiency. When the scheduling algorithm is executed, only the corresponding parameters need to be invoked. Table 3 shows the average CPU time comparison of some benchmarks under the two algorithms.

In the benchmark where the number of work units is equal to 500 (TA111-TA120), the calculation time of SARes is about near to $349.5 \mathrm{~s}$. However, the ISA without knowledgedriven requires close to $934.1 \mathrm{~s}$, which indicates that the knowledge-driven algorithm proposed in this paper is also 
Fig. 5 Algorithm stability analysis

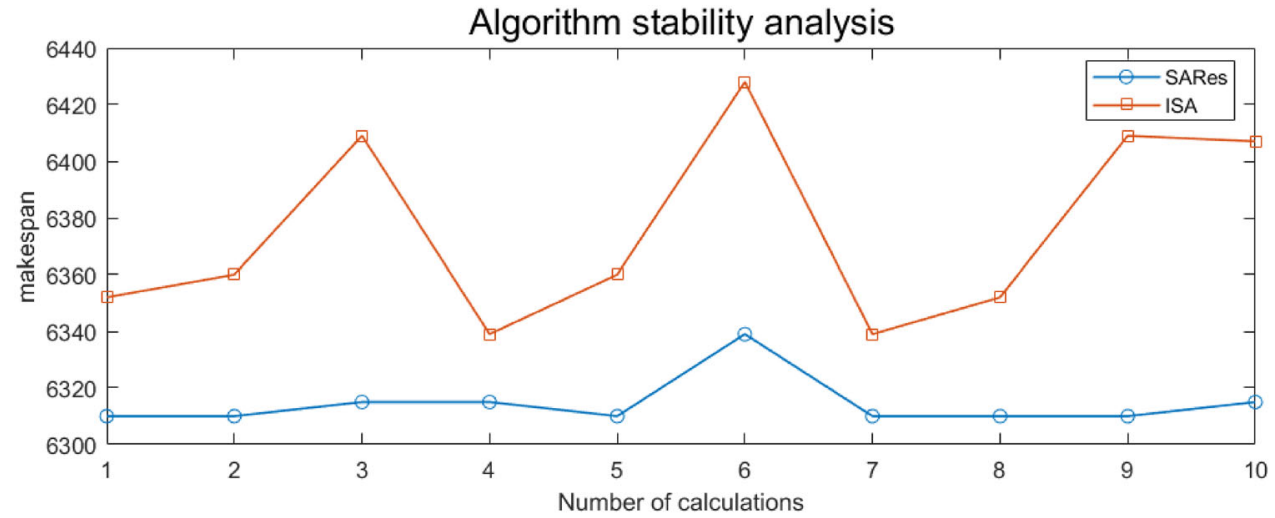

Table 3 Comparison on Taillard benchmark based on the average CPU Time

\begin{tabular}{lllllll}
\hline Problem & $100 * 5$ & $100 * 10$ & $100 * 20$ & $200 * 10$ & $200 * 20$ & $500 * 20$ \\
\hline SARes (s) & $\mathbf{2 0 . 5}$ & $\mathbf{5 2 . 7}$ & $\mathbf{1 1 3 . 5}$ & $\mathbf{1 2 6 . 8}$ & $\mathbf{1 9 7 . 3}$ & $\mathbf{3 4 9 . 5}$ \\
ISA (s) & 70.1 & 94.4 & 180.3 & 231.7 & 455.2 & 934.1 \\
\hline
\end{tabular}

conducive to improving the computational efficiency of the algorithm.

SARes has more knowledge-driven neighborhood search modules than ISA, and its algorithm complexity is bound to increase. But because of this improvement, the algorithm can converge to the local optimal solution more quickly. Both the ISA and SARes algorithms presented in this paper take the unimproved optimal solution for 200 consecutive iterations as the termination condition. Therefore, SARes has a faster computational speed than ISA and it can rapidly converge to the local optimal solution.

\section{ResNet network parameter selection}

There are four commonly used ResNet structures, namely ResNet18, ResNet50, ResNet101 and ResNet152 [8]. These four structures all have their corresponding use environments. Therefore, to determine the network structure parameters in this paper, some benchmarks are selected for experiments and compare their fitting accuracy (Table 4).

Because the data of key blocks in this paper is relatively simple, when the network structure is too complex, there will be an obvious overfitting phenomenon. Combined with the above experiments, it can be known that the most suitable network structure for this paper is ResNet50.

\section{Meta-heuristic algorithm analysis}

To prove that the proposed knowledge-driven neighborhood search algorithm is applicable to any meta-heuristic algorithm, several representative algorithms are selected for
Table 4 Comparison on Taillard benchmark based on the fitting accuracy

\begin{tabular}{lllll}
\hline Problem & ResNet18 & ResNet50 & ResNet101 & ResNet152 \\
\hline $100 * 5$ & $\mathbf{0 . 9 3 1}$ & 0.924 & 0.865 & 0.843 \\
$100 * 10$ & 0.914 & $\mathbf{0 . 9 2 7}$ & 0.885 & 0.897 \\
$100 * 20$ & 0.906 & $\mathbf{0 . 9 3 6}$ & 0.905 & 0.911 \\
$200 * 10$ & 0.912 & $\mathbf{0 . 9 2 5}$ & 0.891 & 0.907 \\
$200 * 20$ & 0.904 & $\mathbf{0 . 9 3 3}$ & 0.904 & 0.913 \\
$500 * 20$ & 0.908 & $\mathbf{0 . 9 3 9}$ & 0.899 & 0.917 \\
ARPD & 0.913 & $\mathbf{0 . 9 3 1}$ & 0.892 & 0.898 \\
\hline
\end{tabular}

comparison, including GA, PSO and IG [21]. Select the TA (TA61-TA120) benchmark as the test case, experimental results are shown in Table 5. Compared with SARes, GARes, PSORes and IGRes in terms of quality, the three algorithms (ISA, GA, PSO, IGRes) are all improved respectively, which proves that the proposed knowledge-driven neighborhood method is effective and can improve the performance of the meta-heuristic algorithm.

\section{Conclusions and future work}

This paper proposes an improved SA algorithm based on the residual network to solve the PFSP problem. This method fuses the knowledge-driven and achieves the-stateof-art results in TA benchmark. At the same time, because the training process of neural network can be completed in advance, it doesn't take up valuable scheduling time.

The main contribution of this paper is to propose the knowledge-driven method to solve the PFSP. According to the experiments, to enhance the optimization capability when solving particular problems, it is very important to incorporate knowledge in intelligent algorithms.

Although the method proposed in this paper has achieved good results, there are still some limitations. First of all, this paper does not prove whether the residual neural network is 
Table 5 Meta-heuristic algorithm analysis

\begin{tabular}{lllllllll}
\hline Problem & \multicolumn{2}{l}{ Relative percentage deviation } \\
\cline { 2 - 8 } & ISA & SARes & GA & GARes & PSO & PSORes & IG & IGRes \\
\hline $100 * 5$ & 0.129 & 0.036 & 0.082 & 0.071 & 0.094 & 0.080 & 0.051 & 0.046 \\
$100 * 10$ & 0.704 & 0.330 & 0.976 & 0.842 & 1.052 & 0.892 & 0.806 & 0.744 \\
$100 * 20$ & 1.978 & 1.584 & 2.823 & 2.011 & 2.658 & 2.455 & 2.347 & 2.291 \\
$200 * 10$ & 0.460 & 0.328 & 0.914 & 0.648 & 0.874 & 0.796 & 0.748 & 0.693 \\
$200 * 20$ & 2.032 & 1.782 & 3.583 & 2.799 & 3.479 & 3.117 & 2.764 & 2.731 \\
$500 * 20$ & 1.515 & 1.189 & 2.892 & 2.314 & 3.017 & 2.697 & 2.541 & 2.533 \\
ARPD & 1.136 & $\mathbf{0 . 8 7 5}$ & 1.878 & $\mathbf{1 . 4 4 8}$ & 1.862 & $\mathbf{1 . 6 7 3}$ & 1.543 & $\mathbf{1 . 5 0 6}$ \\
\hline
\end{tabular}

the most suitable network for this problem. In the future, we can consider adopting other more advanced network structures. Secondly, whenever there is a change in the number of machines in the workshop, we have to retrain the network, which takes a lot of effort. Therefore, in future work, it is necessary to further analyze the relevant network model, improve the theoretical framework, and strive to extend it to all kinds of complex system problems.

Acknowledgements This work was supported by the National Key R\&D Program of China under Grant no. 2019YFB1704600, the National Natural Science Foundation of China under Grant no. 51775216 and 51825502, and in part by the Natural Science Foundation of Hubei Province under Grant no. 2018CFA078.

Open Access This article is licensed under a Creative Commons Attribution 4.0 International License, which permits use, sharing, adaptation, distribution and reproduction in any medium or format, as long as you give appropriate credit to the original author(s) and the source, provide a link to the Creative Commons licence, and indicate if changes were made. The images or other third party material in this article are included in the article's Creative Commons licence, unless indicated otherwise in a credit line to the material. If material is not included in the article's Creative Commons licence and your intended use is not permitted by statutory regulation or exceeds the permitted use, you will need to obtain permission directly from the copyright holder. To view a copy of this licence, visit http://creativecomm ons.org/licenses/by/4.0/.

\section{References}

1. Chen DJ, Lee CY, Hand PC, Mendes P (2007) Parallelizing simulated annealing algorithms based on high-performance computer. J Glob Optim 39(2):261-289

2. Chen SH, Chang PC, Cheng TCE, Zhang Q (2012) A self-guided genetic algorithm for permutation flowshop scheduling problems. Comput Oper Res 39(7):1450-1457

3. Davendra D, Bialic-Davendra M (2013) Scheduling flow shops with blocking using a discrete self-organising migrating algorithm. Int J Prod Res 51(8):2200-2218

4. Ding JY, Song S, Gupta JND, Zhang R, Chiong R, Wu C (2015) An improved iterated greedy algorithm with a Tabu-based reconstruction strategy for the no-wait flowshop scheduling problem. Appl Soft Comput 30:604-613
5. Gao K, Huang Y, Sadollah A, Wang L (2020) A review of energyefficient scheduling in intelligent production systems. Comput IntellSyst 6:237-249

6. Gomez AN, Ren M, Urtasun R, Grosse RB (2017) The reversible residual network: backpropagation without storing activations. Adv Neural Inf Process Syst 30:2214-2224

7. Gui L, Gao L, Li XY (2020) Anomalies in special permutation flow shop scheduling problems. Chin J Mech Eng-En. https://doi.org/1 0.1186/S10033-020-00462-2

8. He K, Zhang X, Ren S, Sun J (2016) Deep residual learning for image recognition. In: Proceedings of the IEEE conference on computer vision and pattern recognition, pp 770-778

9. Johnson SM (1954) Optimal two- and three-stage production schedules with setup times included. Nav Res Logist Q 1(1):61-68

10. Kirkpatrick S, Gelatt CD, Vecchi MP (1983) Optimization by simulated annealing. Science 220(4598):671-680

11. Li X, Gao L (2016) An effective hybrid genetic algorithm and tabu search for flexible job shop scheduling problem. Int J Prod Econ 174:93-110

12. Li X, Yin M (2013) An opposition-based differential evolution algorithm for permutation flow shop scheduling based on diversity measure. Adv Eng Softw 55:10-31

13. Li XY, Lu C, Gao L, Xiao SQ, Wen L (2018) An effective multiobjective algorithm for energy efficient scheduling in a real-life welding shop. IEEE Trans Ind Inform 14(12):5400-5409

14. Li JC, Fang FM, Mei KF, Zhang GX (2018) Multi-scale residual network for image super-resolution. In: Proceedings of the European conference on computer vision, pp 517-532

15. Lin L, Yuan H, Guo LR (2018) Optimization method of residual networks of residual networks for image classification. Int Conf Int Comput 10956:212-222

16. Liu YH, Cao BY, Li HH (2020) Improving ant colony optimization algorithm with epsilon greedy and Levy flight. Comput IntellSyst. https://doi.org/10.1007/s40747-020-00138-3

17. Liu Y, Yin M, Gu W (2014) An effective differential evolution algorithm for permutation flow shop scheduling problem. Appl Math Comput 248:143-159

18. Liu ZF, Yan J, Cheng Q, Yang CB, Sun SW, Xue D (2020) The mixed production mode considering continuous and intermittent processing for an energy-efficient hybrid flow shop scheduling. J Clean Prod 119071:1-17

19. Mhapsekar M, Mhapsekar P, Mhatre A, Sawant V (2020) Implementation of residual network (ResNet) for devanagari handwritten character recognition. In: Vasudevan H, Michalas A, Shekokar N, Narvekar M (eds) Advanced computing technologies and applications. Algorithms for intelligent systems. Springer, Singapore, pp 137-148. https://doi.org/10.1007/978-981-15-3242-9_14

20. Rahman HF, Sarker R, Essam D (2017) A real-time order acceptance and scheduling approach for permutation flow shop problems. Oper Res 57(4):345-347 
21. Ruiz R, Stützle T (2007) A simple and effective iterated greedy algorithm for the permutation flowshop scheduling problem. Eur $\mathbf{J}$ Oper Res 177(3):2033-2049

22. Sayed GI, Hassanien AE (2018) A hybrid SA-MFO algorithm for function optimization and engineering design problems. Comput IntellSyst 4:195-212

23. Shao W, Pi D (2016) A self-guided differential evolution with neighborhood search for permutation flow shop scheduling. Expert SystAppl 51:161-176

24. Sun BQ, Wang L (2020) An estimation of distribution algorithm with branch-and-bound based knowledge for robotic assembly line balancing. Comput IntellSyst. https://doi.org/10.1007/s40747-02 0-00166-Z

25. Suresh S, Anima N (2016) Social group optimization (SGO): a new population evolutionary optimization technique. Comput IntellSyst 2:173-203

26. Valeryi MB, Stanislav AK, Liudmyla SK (2020) Galois field augmentation model for training of artificial neural network in dentistry data-Ce. Bus Appl 48:339-369

27. Wang GC, Gao L, Li XY, Li PG, Tasgetiren MF (2020) Energyefficient distributed permutation flow shop scheduling problem using a multi-objective whale swarm algorithm. Swarm Evol Comput 57:100716

28. Wang JJ, Wang L (2020) A knowledge-based cooperative algorithm for energy-efficient scheduling of distributed flow-shop. IEEE Trans Syst Man CybernSyst 50(5):1805-1819

29. Wang L, Wang S, Zheng X (2016) A hybrid estimation of distribution algorithm for unrelated parallel machine scheduling with sequence-dependent setup times. IEEE/CAA J AutomSinica 3(3):235-246

30. Wang SY, Wang L (2016) An estimation of distribution algorithmbased memetic algorithm for the distributed assembly permutation flow-shop scheduling problem. IEEE Trans Syst Man CybernSyst 46(1):139-149

31. Wang YH, Chen WY (2019) A decomposition-based hybrid estimation of distribution algorithm for practical mean-cvar portfolio optimization. In: Proceedings of the international conference on intelligent computing, Nanchang, China, pp 38-50

32. Wu C, Wang L (2018) A multi-model estimation of distribution algorithm for energy efficient scheduling under cloud computing system. J Parallel Distr Com 117:63-72

33. Rafajłowicz W (2015) A hybrid differential evolution-gradient optimization method. In: Proceedings of the international conference on artificial intelligence and soft computing, pp 379-388
34. Xie Z, Zhang C, Shao X, Lin W, Zhu H (2014) An effective hybrid teaching-learning-based optimization algorithm for permutation flow shop scheduling problem. Adv Eng Softw 77:35-47

35. Yenisey MM, Yagmahan B (2014) Multi-objective permutation flow shop scheduling problem: literature review, classification and current trends. Comput Hum Behav 45:119-135

36. Yin LJ, Li XY, Gao L, Lu C, Zhang Z (2017) A novel mathematical model and multi-objective method for the low-carbon flexible job shop scheduling problem, considering productivity, energy efficiency and noise reduction. Sustain Comput Inf 13:15-30

37. Yoshua B, Andrea L, Antoine P (2020) Machine learning for combinatorial optimization: a methodological Tour d'Horizon. Eur J Oper Res. https://doi.org/10.1016/j.ejor.2020.07.063

38. Yuan H (2019) Spatiotemporal task scheduling for heterogeneous delay-tolerant applications in distributed green data centers. IEEE/CAA J Autom Sin 16(4):1686-1697

39. Zheng XL, Wang L (2018) A collaborative multiobjective fruit fly optimization algorithm for the resource constrained unrelated parallel machine green scheduling problem. IEEE Trans Syst Man CybernSyst 48(5):790-800

40. Zhong ZL (2017) Spectral-spatial residual network for hyperspectral image classification: a 3-D deep learning framework. IEEE Trans Geosci Remote Sens 56(2):847-858

Publisher's Note Springer Nature remains neutral with regard to jurisdictional claims in published maps and institutional affiliations 